\title{
INRG Stage L2
}

National Cancer Institute

\section{Source}

National Cancer Institute. INRG Stage L2. NCI Thesaurus. Code C133429.

Locoregional neuroblastoma with one or more image defined risk factors (IDRFs). 